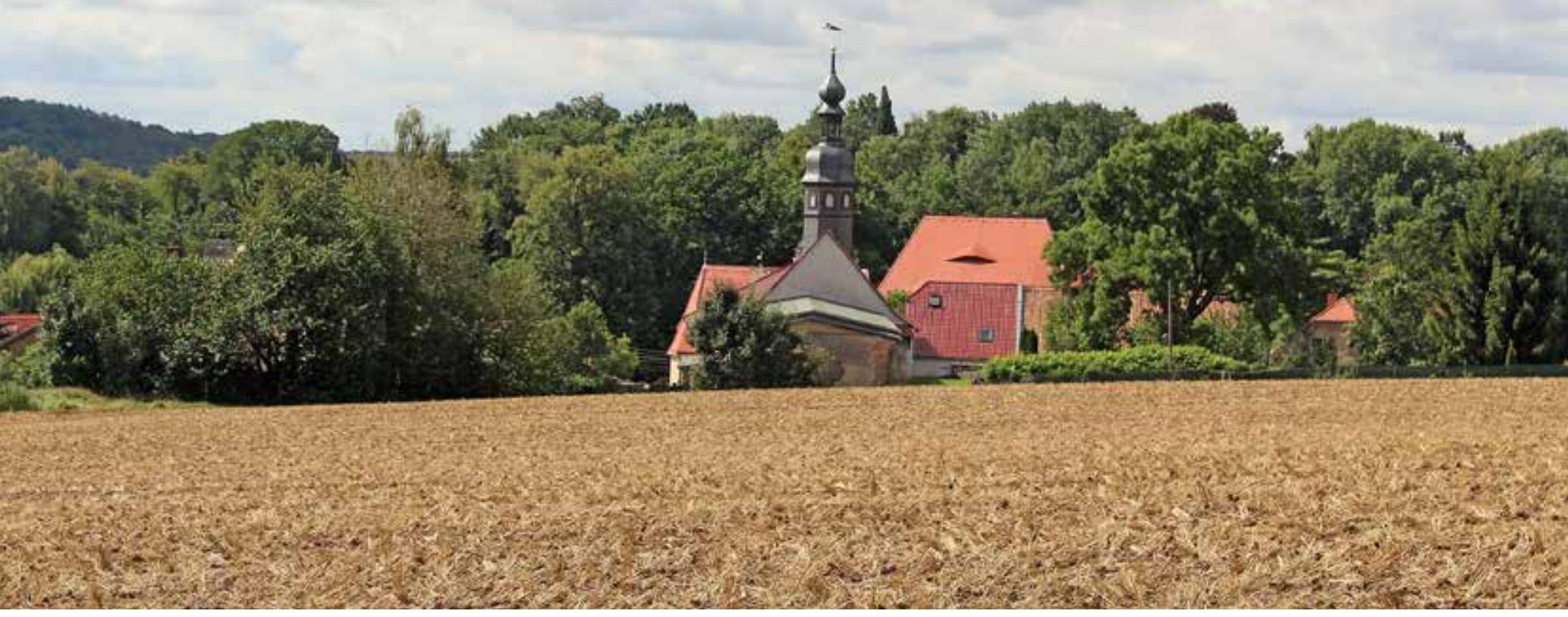

\title{
Der Förderverein für Heimat und Kultur in der Lommatzscher Pflege und das LEADER-Gebiet Lommatzscher Pflege
}

\author{
Michaela Stock, Marcel Borisch
}

Die sanften Hügel der Lommatzscher Pflege erstrecken sich links der Elbe zwischen den Städten Dresden und Meißen im Osten, Wilsdruff und Nossen im Süden, Döbeln und Mügeln im Westen sowie Oschatz und Riesa im Norden. In der Mitte liegt die namensgebende kleine Stadt Lommatzsch. Das Gebiet kreuzen die Bundesstraßen B 6, B 101 und B 169. Die Autobahnen A 4 und A 14 rahmen das Gebiet ein und bilden die Anknüpfungspunkte an den überregionalen Verkehr. Diese guten infrastrukturellen Voraussetzungen, die Nähe zu den Großstädten mit ihren Kulturangeboten und die gute soziale Infrastruktur mit Kinderkrippen, Kindergärten,
Grund- und Oberschulen machen die Region für Neuansiedlungen von Bewohnern und Wirtschaftsunternehmen interessant. Voll erschlossene Gewerbegebiete stehen in den Gemeinden hierfür zur Verfügung.

Die wegen ihrer fruchtbaren Böden als Kornkammer Sachsens bekannte Region besitzt dank einer gemeinsamen Historie, bezeugt durch kulturlandschaftliche Einheit und Baukultur eine starke regionale Identität. Charakteristisch für die Lommatzscher Pflege ist die waldarme Landschaft mit den darin eingebetteten zahlreichen kleinen Ortschaften. Seit Jahrtausenden sorgen die guten Böden für hohe Er-
Blick zum Rittergut Schleinitz. Schleinitz ist eines von vielen Dörfern im Herzen des LEADERGebiets Lommatzscher Pflege Foto: Matthias Donath 
1 LEADER heißt auf Französisch: „Liason entre actions de développement de l'èconomie rurale“. Übersetzt bedeute das „Verbindung zwischen Aktionen zur Entwicklung der ländlichen Wirtschaft" Dieses Projekt ist eine Gemeinschaftsinitiative der Europäischen Union, mit der seit 1991 modellhaft innovative Aktionen im ländlichen Raum gefördert werden. Lokale Aktionsgruppen erarbeiten mit den Akteuren vor Ort maßgeschneiderte Entwicklungskonzepte für ihre Region aus. Ziel ist es, die ländlichen Regionen Europas auf dem Weg zu einer eigenständigen Entwicklung zu unterstützen.

Lage und Abgrenzung des LEADER-Gebiets Lommatzscher Pflege träge. Große Drei- und Vierseithöfe weisen auf diese lange landwirtschaftliche Tradition hin. Aber auch traditionelles Handwerk ist verbreitet und bietet der Bevölkerung Arbeitsplätze.

Der Zusammenschluss von zehn Gemeinden zum Förderverein für Heimat und Kultur in der Lommatzscher Pflege e.V. im Jahr 1998 war der erste wichtige Schritt für eine enge Zusammenarbeit in der Region. Der Förderverein richtet sein Hauptaugenmerk auf die Vernetzung der Region und der Pflege des Heimatgedankens, indem den Bürgern die geschichtliche und gegenwertige Entwicklung der Lommatzscher Pflege in Kunst, Kultur und Landschaft nahe gebracht wird. Die Stärkung der eigenen Identität der Menschen in der Lommatzscher Pflege gehört zu den wichtigsten Ansatzpunkten, um ihnen eine stabile Basis für Veränderungsprozesse zu geben. Nur wenn sich Bürger und Unternehmer in Ihrer Heimat identifizieren, ihre Werte kennen und teilen, werden sie sich für die zukünftigen Aufgaben der Region engagieren.

Seit dem Jahr 2005 stellt sich der Förderverein deutlich den Problemen des Wandels in der Lommatzscher Pflege. Der Verein entwickelte mit zahlreichen weiteren Akteuren der Lommatzscher Pflege ein Integriertes Entwicklungskonzept (ILEK). Mit diesem Konzept wurde die Region als LEADER-Gebiet ${ }^{1}$ für die Jahre 2007 bis 2013 ernannt. Über 22 Millionen Euro europäischer Fördermittel flossen in die zehn Gemeinden der Lommatzscher Pflege zur Umsetzung privater und öffentlicher Maßnahmen.

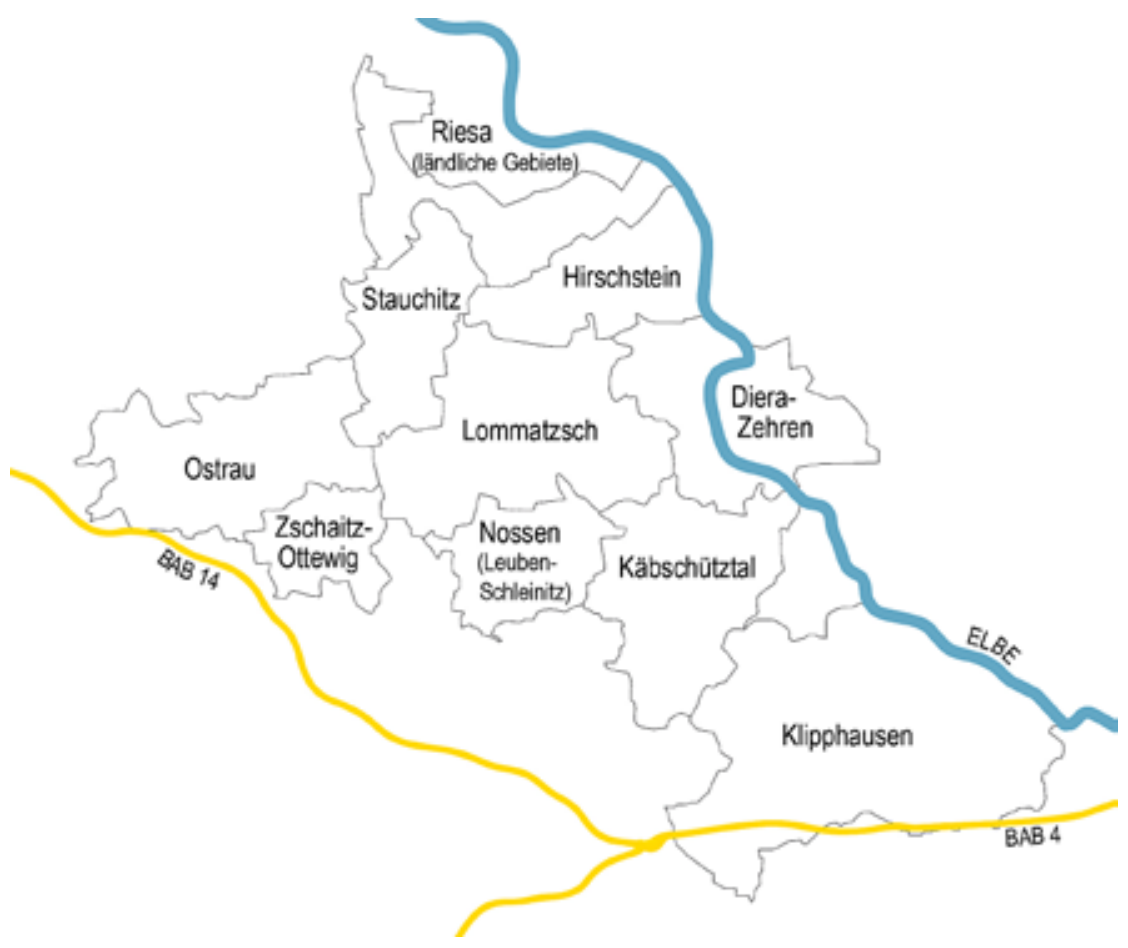

In Vorbereitung der Förderperiode von 2014 bis 2020 hat der Förderverein für Heimat und Kultur in der Lommatzscher Pflege e.V. als Träger der Lokalen Aktionsgruppe (LAG) des LEADERGebietes Lommatzscher Pflege die Interessen der Akteure zusammengeführt und Ziele einer zukünftigen Entwicklung diskutiert. Unter der aktiven Mitarbeit von Bürgern, Vereinen, Unternehmen und Kommunen entstand die LEADEREntwicklungsstrategie (LES). Sie war die Grundlage dafür, dass im LEADER-Gebiet erneut Fördermittel ausgegeben werden können. Die Europäische Union und der Freistaat Sachsen stellen zur Umsetzung der LEADER-Entwicklungsstrategie Fördermittel aus dem europäischen Fonds ELER (Entwicklungsprogramm für den ländlichen Raum im Freistaat Sachsen) und dem sächsischen Fachprogramm EPLR (Europäischer Landwirtschaftsfonds für die Entwicklung des ländlichen Raums) bereit.

Im April 2015 wurde die Lommatzscher Pflege erneut zum LEADER-Gebiet ernannt. Das Regionalmanagement für das LEADER-Gebiet liegt nunmehr direkt in den Händen des Fördervereins für Heimat und Kultur in der Lommatzscher Pflege e.V. Das LEADER-Gebiet umfasst sieben Landgemeinden (Diera-Zehren, Hirschstein, Käbschütztal, Klipphausen, Ostrau Stauchitz und Zschaitz-Ottewig) und die Stadt Lommatzsch mit ihren zugehörigen Ortsteilen, die nun zur Stadt Nossen gehörenden Ortsteile der ehemaligen Gemeinden Leuben-Schleinitz und Ketzerbachtal sowie die ländlichen Ortsteile der Stadt Riesa. Das Gebiet umfasst eine Fläche von etwa 464 Quadratkilometern, in der ca. 36.000 Einwohner leben.

Das Leitbild der Lommatzscher Pflege lautet: „Die Lommatzscher Pflege bietet ihrer Bevölkerung attraktive Arbeits- und Lebensperspektiven und wird die Auswirkungen des demografischen Wandel im ländlichen Raum aktiv gestalten. Grundlage dafür sind die nachhaltigen Potenziale einer gewachsenen Region mit großer historischer Bedeutung für Sachsen, wertvollen Böden, traditionellen Handwerk und innovativen Unternehmen, der charakteristischen Kulturlandschaft und ihren typischen Höfen und Dörfern. Die wertvollen Potenziale bilden zusammen mit den Chancen aus der Nähe zu den prosperierenden Wirtschaftszentren Sachsens sowie dem hohen Engagement der Bürger die Vorrausetzung zum Erreichen der gesteckten Ziele.“ Aus dem Leitbild leitet sich das Slogan ab: „Wo Werte Wachsen.“

Die Fördervorhaben sollen dazu beitragen, Einwohner in der Lommatzscher Pflege zu halten und die Kulturlandschaft lebenswerter und liebenswerter zu gestalten. Die Projekte im Rahmen 


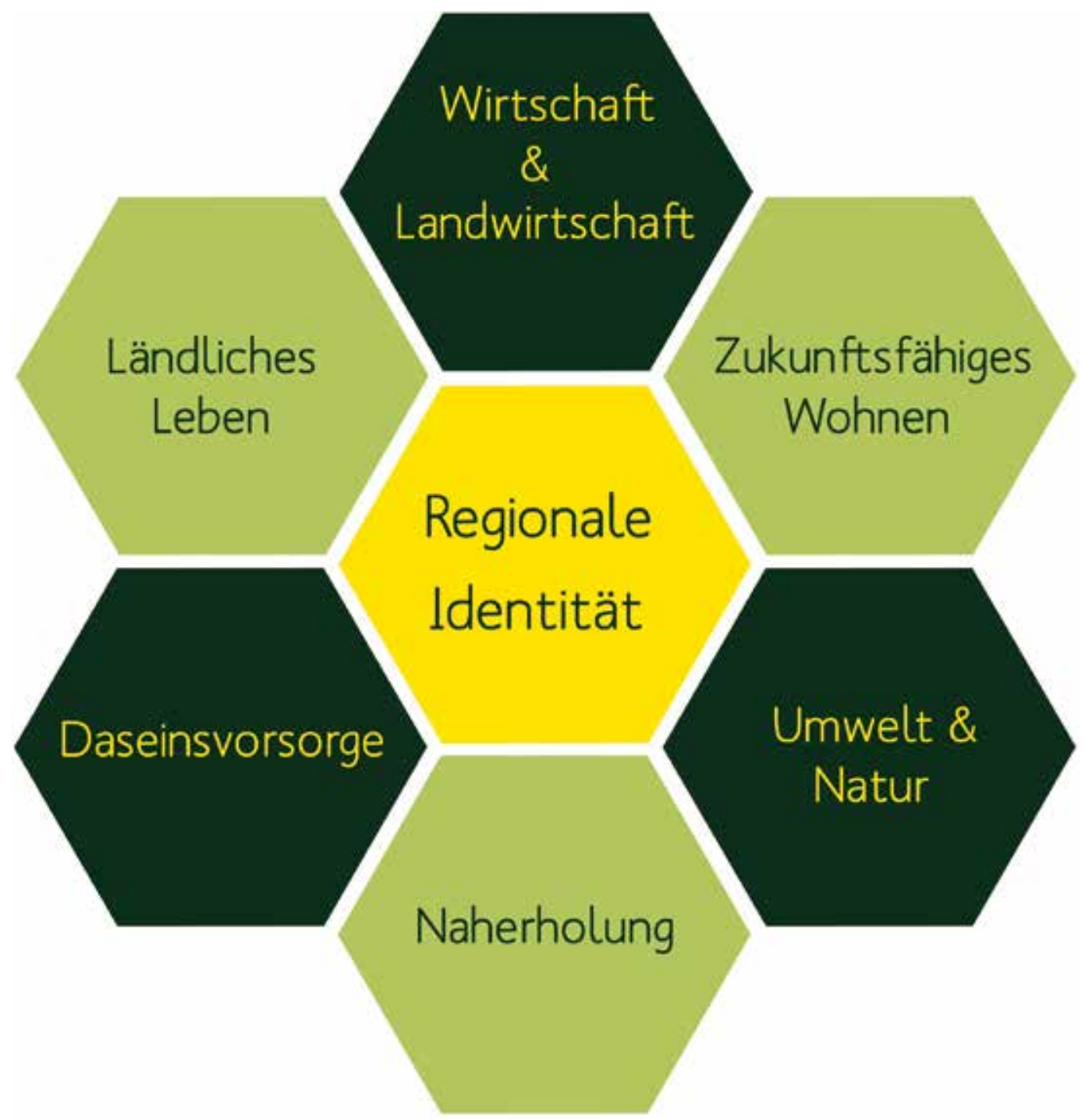

der LEADER-Entwicklungsstrategie setzen eine intensive Zusammenarbeit der politischen Entscheidungsträger der Region voraus. Dies erfolgt in einem Entscheidungsgremium gemeinsam mit ehrenamtlichen Bürgern der Region sowie im Vorstand des Fördervereins für Heimat und Kultur in der Lommatzscher Pflege e.V. Hierdurch werden allmählich der Gedanke der ganzheitlichen Entwicklung der Region gestärkt und Konkurrenzängste der Gemeinden abgebaut.

Zahlreiche regionale und überregionale Veranstaltungen prägen das kulturelle Leben in der Lommatzscher Pflege und tragen zur Identität der Region bei. Die Organisation und Durchführung wird von ehrenamtlich Engagierten durchgeführt. Der Verein unterstützt das kulturelle Leben in der Lommatzscher Pflege. Neben sportlichen Veranstaltungen führt der Verein verschiedene Wettbewerbe durch. Die Öffentlichkeitsarbeit konnte in den letzten Jahren ausgebaut werden.

Die Antragstellung für LEADER-Fördermittel wird dabei durch themenbezogene Projekt-Auf- rufe gestartet. Von der Um- und Wiedernutzung ländlicher Bausubstanz zu Wohnzwecken bis zur Entwicklung und/oder Etablierung regionaler Produkte besteht eine große Bandbreite an möglichen Förderthemen. Rund zehn Millionen Euro hat die Region dafür bis 2020 zur Verfügung. Für Vorhaben, die der Umsetzung der Strategie des LEADER-Gebietes Lommatzscher Pflege dienen, besteht somit die Möglichkeit auf

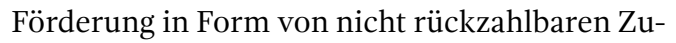
schüssen (Anteilsfinanzierung). Die Strategie setzt sich aus einem übergeordneten Leitbild, sieben Handlungsfeldern und sieben LES-relevanten strategischen Zielen zusammen. Diese strategischen Ziele sollen die wichtigen Entwicklungsrichtungen der Region Lommatzscher Pflege abbilden, die im Rahmen von LEADER vorgenommen werden.

Alle notwendigen Unterlagen und Informationen stehen Ihnen im Internet unter www. lommatzscher-pflege.de unter dem Menüpunkt „Förderung“ zur Verfügung.
Handlungsfelder der Lokalen Entwicklungsstrategie für das LEADER-Gebiet Lommatzscher Pflege

\section{Autoren}

Marcel Borisch, Michaela Stock Regionalmanagement LEADER-Gebiet Lommatzscher Pflege Neugasse 39/40 01662 Meißen 